\title{
Primeiro registro de Myotis albescens (É. Geoffroy, 1806) (Chiroptera, Vespertilionidae) para o Estado do Paraná, Brasil
}

\author{
João Marcelo Deliberador Miranda ${ }^{1,3}$, Atenisi Pulchério-Leite ${ }^{1}$, Itiberê Piaia Bernardi ${ }^{2}$ \& \\ Fernando de Camargo Passos ${ }^{1}$ \\ Biota Neotropica $v 7(n 1)$ \\ http://www.biotaneotropica.org.br/v7n1/pt/abstract?short-communication+bn03407012007 \\ Recebido em 20/06/06 \\ Versão reformulada recebida em 13/12/06 \\ Publicado em 28/03/07 \\ ${ }^{1}$ Laboratório de Biodiversidade, Conservação e Ecologia de Animais Silvestres, Departamento de \\ Zoologia, Universidade Federal do Paraná, CP 19020, Curitiba, PR, Brasil, \\ ${ }^{2}$ Rua Guerino Cerutti, 161, CEP 98400-000, Frederico Westphalen, RS. \\ ${ }^{3}$ Autor para correspondência: João Marcelo Deliberador Miranda, e-mail: guaribajoao@yahoo.com.br \\ http://zoo.bio.ufpr.br/mamifero/index.htm
}

\begin{abstract}
Miranda, J.M.D., Pulchério-Leite, A., Bernardi, I.P. \& Passos, F.C. First record of Myotis albescens (É. Geoffroy) from Paraná State, Brazil (Chiroptera, Vespertilionidae). Biota Neotrop. Jan/Apr 2007 vol. 7 , no. 1 www.biotaneotropica.org.br/v7n1/pt/abstract?short-communication+bn03407012007 ISSN 1676-0603.

This is the first record of the vespertilionid bat Myotis albescens (É. Geoffroy, 1806) from Paraná State. An individual of $M$. albescens was recorded on Mutum Island, Porto Rico Municipality (22 $46^{\prime} 20^{\prime \prime} \mathrm{S}$ and $\left.53^{\circ} 16^{\prime} 01^{\prime \prime} \mathrm{W}\right)$. This record fills an important gap in the distribution of M. albescens, as well as contributes to the knowledge of the mammals of Paraná.

Keywords: bat distribution, mammals of Paraná.

\section{Resumo}

Miranda, J.M.D., Pulchério-Leite, A., Bernardi, I.P. \& Passos, F.C. Primeiro registro de Myotis albescens (É. geoffroy, 1806) (Chiroptera, vespertilionidae) para o Estado do Paraná, Brasil. Biota Neotrop. Jan/ Apr 2007 vol. 7, no. 1 www.biotaneotropica.org.br/v7n1/pt/abstract?short-communication+bn03407012007 ISSN 1676-0603.

Este é o primeiro registro do morcego vespertilionídeo Myotis albescens (É. Geoffroy, 1806) para o Estado do Paraná. Um indivíduo de Myotis albescens foi registrado na Ilha Mutum, Município de Porto Rico $\left(22^{\circ} 46^{\prime} 20^{\prime}\right.$ 'S e $53^{\circ} 16^{\prime} 01^{\prime}$ W). Esse registro preenche parte de uma lacuna na distribuição de M. albescens, além de contribuir com o conhecimento da mastofauna paranaense.
\end{abstract}

Palavras-chave: distribuição de morcegos, mamíferos do Paraná. 


\section{Introdução}

Myotis albescens (É. Geoffroy, 1806) é um morcego de pequeno porte (4 - $11 \mathrm{~g})$, de dieta insetívora como a maioria dos Vespertilionidae (LaVal 1973, Barquez et al. 1999, Achaval et al. 2004). Sua distribuição geográfica vai desde o sul do México até a Argentina e Uruguai (Acosta y Lara 1950, Cabrera 1957, LaVal 1973, Koopman 1993, Nowak 1994, Simmons 2005), passando por grande parte do território brasileiro (Marinho-Filho 1996a, b). Os únicos registros para a região Sul do Brasil são de dois espécimes procedentes do Estado do Rio Grande do Sul, onde se encontra na Lista Vermelha da Fauna Ameaçada sob a categoria Dados Insuficientes (Pacheco \& Freitas 2003). Não há registro de $M$. albescens para os Estados do Paraná e de Santa Catarina (Miretzki 2003, Cherem et al. 2004, Peracchi et al. 2006). Assim, a presente comunicação tem por objetivo registrar a ocorrência de $M$. albescens no Estado do Paraná, contribuindo para preencher uma lacuna em sua distribuição, bem como para o conhecimento da mastofauna estadual.

\section{Material e Métodos}

O registro de Myotis albescens foi baseado em um espécime coletado durante um levantamento de morcegos realizado na Ilha Mutum (Alto Rio Paraná), Município de Porto Rico, região noroeste do Paraná (22 $46^{\prime} 20^{\prime \prime} \mathrm{S}$ e $53^{\circ} 16^{\prime} 01^{\prime}$ 'W) (Figura 1). O exemplar foi identificado com base nos caracteres diagnósticos da espécie segundo vários autores (LaVal 1973, Vizotto \& Taddei 1973, Barquez et al. 1999, López-González et al. 2001). As medidas do exemplar foram aferidas segundo os critérios indicados por Barquez et al. (1999).

A Ilha Mutum pertence ao arquipélago fluvial do Alto Rio Paraná, pertencendo à formação da Floresta Estacional Semidecidual Aluvial (Campos \& Souza 1997) e contando com aproximadamente 1050 ha. A área é formada por floresta primária alterada, mata ciliar e floresta secundária (em vários estágios de sucessão florestal), além de ambientes alterados (Campos \& Souza 1997). O clima da região é Cfa h segundo Köeppen, com temperaturas anuais médias de $22{ }^{\circ} \mathrm{C}$ e altidude de $250 \mathrm{~m}$ (Maack 1968). A Ilha Mutum se encontra na Área de Proteção Ambiental Federal das Ilhas e Várzeas do Rio Paraná (Mikich \& Bérnils 2004).

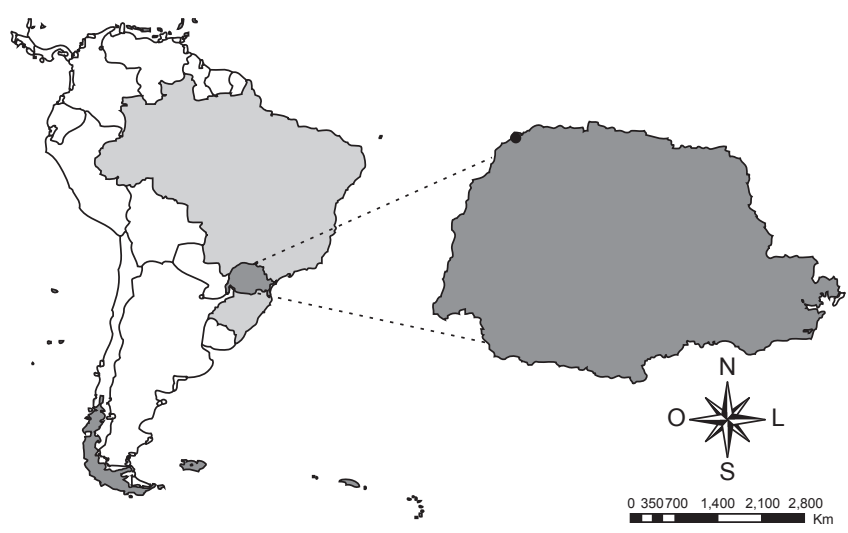

Figura 1. Mapa da América do Sul, destacando o Brasil e o Estado do Paraná, e a localização da Ilha Mutum, Município de Porto Rico (22 46' $20^{\circ}$ ' S e $53^{\circ} 16^{\prime} 01$ ' W), Estado do Paraná.

Figure 1. Map of South America, highlighting Brazil and Paraná State, and the location of the Mutum Island, Municipality of Porto Rico (22 $46^{\prime} 20^{\prime \prime} \mathrm{S}$ and $\left.53^{\circ} 16^{\prime} 01^{\prime \prime} \mathrm{W}\right)$, Paraná State.

\section{Resultados e Discussão}

Em 13 de outubro de 2005 um exemplar de M. albescens macho adulto foi coletado com rede de neblina ("mist net") na Ilha Mutum. Esse exemplar foi capturado às 19 horas em um local de floresta secundária, próximo a uma habitação humana. O exemplar está tombado na Coleção Científica de Mastozoologia da Universidade Federal do Paraná (DZUP-CCMZ) sob o número tombo DZUPCCMZ 226.

Embora a ocorrência de $M$. albescens para o Paraná fosse esperada com base na proposta biogeográfica de Marinho-Filho (1996b), até o presente momento não existiam registros formais do táxon segundo Mireztki (2003) e demais trabalhos recentes (Sekiama et al. 2001, Reis et al. 2000 e 2003, Bianconi et al. 2004, Peracchi et al. 2006, Tavares et al. no prelo). O exemplar foi seguramente identificado apresentando os caracteres diagnósticos da espécie (LaVal 1973, Vizotto \& Taddei 1973, Barquez et al. 1999, López-González et al. 2001). Dentre esses, destacam-se alguns caracteres qualitativos, tais como: plagiopatágio inserido na base dos artelhos; trago de largura igual ao longo de seu comprimento, com o lobo basal pouco desenvolvido; coloração geral cinzenta agrisalhada, ventre grisalho esbranquiçado, especialmente a região perianal; uropatágio com borda pigmentada e com franja de pêlos; pés grandes e arredondados. Além desses caracteres externos, destaca-se também o crânio que não possui crista sagital e apresenta o segundo par de pré-molares superiores alinhados aos outros dentes e não deslocados para a face lingual. As medidas cranianas do exemplar foram: comprimento condilobasal 13,4 $\mathrm{mm}$; largura interorbital 5,0 mm; comprimento total do crânio $14,1 \mathrm{~mm}$; largura da constrição pósorbitária 4,1 mm; largura da caixa craniana 7,3 mm; comprimento da série de dentes superiores $5,1 \mathrm{~mm}$; largura mastoidal $7,5 \mathrm{~mm}$; série de dentes inferiores 5,2 mm; comprimento da mandíbula 10,0 mm; largura entre caninos superiores $3,7 \mathrm{~mm}$; largura entre os molares superiores 5,6 mm. Esse exemplar diferencia-se de $M$. nigricans (Schinz, 1821) pelo maior tamanho e coloração; diferencia-se de M. ruber (Geoffroy, 1806), M. keaysi (Allen, 1914), M. simus (Thomas, 1901) e M. riparius (Handley, 1960) por não apresentar crista sagital e pelo alinhamento dos segundos pré-molares superiores em relação aos outros dentes; e separa-se de M. levis (Geoffory, 1824) pela maior largura da constrição pós-orbitária e pelo menor comprimento da série de dentes inferiores (Barquez et al. 1999, López-González et al. 2001).

Miretzki (2003) considerou a região noroeste do Paraná, onde se encontra a Ilha Mutum como de média prioridade para estudos de morcegos. O presente registro, além de outros também recentes (Miranda et al. 2006a e 2006b), confirma a carência de estudos no Estado do Paraná. Essa espécie não constou da Lista Vermelha de Espécies Ameaçadas no Paraná (Margarido \& Braga 2004). Tendo em vista que alguns dos critérios fundamentais para definir espécies ameaçadas são: a distribuição, o número de registros, presença e situação em unidades de conservação (Gärdenfors et al. 2001, IUCN 2001), sugerimos que essa espécie conste em uma posterior revisão dessa lista, categorizada como Dados Insuficientes, como classificada para o Rio Grande do Sul (Pacheco \& Freitas 2003).

\section{Agradecimentos}

Agradecemos ao CNPq pelas Bolsas de estudos de JMDM e de APL. À Secretaria Estadual de Saúde do Estado do Paraná - SESA/PR pelo apoio logístico na região de Porto Rico. Aos sócios do Clube Rio Baía pela autorização para utilizar as dependências do clube na Ilha Mutum. A Maria Fernanda M. Azevedo-Barros pelo auxílio em campo. Aos Srs. Adílson B. Secorum e Edílson C. Colhera, pelo apoio técnico e logístico. 


\section{Referências Bibliográficas}

ACHAVAL, F., CLARA, M. \& OLMOS, A. 2004. Mamíferos de la República Oriental del Uruguay. Imprimex, Montevideo.

ACOSTA Y LARA, E.F. 1950. Qirópteros del Uruguay. Comunicaciones Zoologicas del Museo de Historia Natural de Motevideo 58(3):1-73.

BARQUEZ, R.M., MARES, M.A. \& BRAUN, J.K. 1999. The Bats of Argentina. Special Publications. Museum of Texas Tech University 42:1-275.

BIANCONI, G.V., MIKICH, S.B. \& PEDRO, W.A. 2004. Diversidade de morcegos (Mammalia, Chiroptera) em remanescentes florestais do município de Fênix, noroeste do Paraná, Brasil. Revista Brasileira de Zoologia 21(4):943-954.

CABRERA, A. 1957. Catalogo de los Mamiferos de America del Sur. Museo Argentino de Ciencias Naturales Bernardino Rivadavia e Instituto Nacional de Investigación de las Ciencias Naturales 4(1):01-307.

CAMPOS, J.B. \& SOUZA, M.C. 1997. Vegetação. In A Planície de Inundação do Alto Rio Paraná. Aspectos físicos, biológicos e socioeconômicos (A.E.A M. Vazzoler, A.A. Agostinho \& N.S. Hahn, eds.). EDUEM/ UEM-Nupélia, Maringá, p.331-342.

CHEREM, J.J., SIMÕES-LOPES, P.C., ALTHOFF, S. \& GRAIPEL, M.E. 2004. Lista dos Mamíferos do Estado de Santa Catarina, Sul do Brasil. Mastozoología Neotropical 12(2):151-184.

GÄRDENFORS, U., HILTON-TAYLOR, C., MACE, G.M. \& RODRIGUEZ, J.P. 2001. The application of IUCN red list criteria at regional levels. Conservation Biology 15(5):1206-1212

IUCN. 2001. IUCN Red List Categories and Criteria Version 3.1. Cambridge, International Union for Conservation of Nature and Natural Resources - Species Survival Commision, Washington.

KOOPMAN, K.F. 1993. Order Chiroptera. In Mammal Species of the World, a taxonomic and geographic reference (D.E. WILSON \& D. REEDER, eds.). Smithsonian Institution Press, Washington, p.137-241.

LAVAL, R. 1973. A revision of the Neotropical bats of the genus Myotis Natural History Museum Los Angeles County 15:1-54.

LÓPEZ-GONZÁLEZ, C., PRESLEY, S.J., OWEN, R.D. \& WILLIG, M.R. 2001. Taxonomic status of Myotis (Chiroptera: Vespertilionidae) in Paraguay. Journal of Mammalogy 82(1):138-160.

MAACK, R. 1968. Geografia física do Estado do Paraná. Max Roesner, Curitiba.

MARINHO-FILHO, J. 1996a. The Brazilian cerrado bat fauna and its conservation. Chiroptera Neotropical 2(1):37-39.

MARINHO-FILHO, J. 1996b. Distribution of bat diversity in the Southern and Southeastern Brazilian Atlantic Forest. Chiroptera Neotropical 2(2):51-54

MARGARIDO, T.C.C. \& BRAGA, F.G. 2004. Mamíferos. In Livro vermelho da fauna ameaçada no Estado do Paraná (S.B. MIKICH \& R.S. BÉRNILS, eds.). Governo do Paraná, Curitiba, p.27-142.

MIKICH, S.B. \& BÉRNILS, R.S. 2004. Livro vermelho da fauna ameaçada no Estado do Paraná. Governo do Paraná, Curitiba.

MIRANDA, J.M.D., BERNARDI, I.P. \& PASSOS, F.C. 2006. A new species of Eptesicus (Mamalia: Chiroptera: Vespertilionidae) from the Atlantic forest Brazil. Zootaxa 1383: 57-68.

MIRANDA, J.D.M., PULCHÉRIO-LEITE, A., MORO-RIOS, R.F. \& PASSOS, F.C. 2006. Primeiro registro de Histiotus montanus (Philippi \& Landbeck) para o Estado do Paraná, Brasil (Chiroptera, Vespertilionidae). Revista Brasileira de Zoologia 23(2):584-587.
MIRETZKI, M. 2003. Morcegos do Estado do Paraná, Brasil (Mammalia, Chiroptera): riqueza de espécies, distribuição e síntese do conhecimento atual. Papéis Avulsos de Zoologia 43(6):101-138.

NOWAK, R.M. 1994. Walker's Bats of the World. Johns Hopkins University Press, Baltimore.

PACHECO, S.M. \& FREITAS, T.R.O. 2003. Quirópteros. In Livro vermelho da fauna ameaçada de extinção no Rio Grande do Sul (C.S. FONTANA, G.A. Bencke \& R.E. Reis, eds.). EDIPUCRS, Porto Alegre, p.493-497.

PERACCHI, A.L., LIMA, I.P., REIS, N.R., NOGUEIRA, M.R. \& FILHO, H.O. 2006. Ordem Chiroptera. In Mamíferos do Brasil (N.R. REIS, A.L. PERACCHI, W. A. PEDRO \& I. P. LIMA, eds.). Governo do Paraná/ SEMA/SBZ, Curitiba, p.155-234.

REIS, N.R., PERACCHI, A.L., SEKIAMA, M.L. \& LIMA, I.P. 2000. Diversidade de morcegos (Chiroptera, Mammalia) em fragmentos florestais no estado do Paraná, Brasil. Revista Brasileira de Zoologia 17(3):697-704

REIS, N.R., PERACCHI, A.L. \& LIMA, I.P. 2003. Morcegos da bacia do rio Tibagi. In A Bacia do Rio Tibagi. (M.E. Medri, E. Bianchini, O.A. Shibatta \& J.A. Pimenta, eds.). UEL/ KLABIN/ Fundação Araucária/ Sercontel/ Confepar, Londrina, p.251-270.

SEKIAMA, M.L., REIS, N.R., PERACCHI, A.L. \& ROCHA, V.J. 2001. Morcegos do Parque Nacional do Iguaçu, Paraná (Chiroptera, Mammalia). Revista Brasileira de Zoologia 18(3):749-754.

SIMMONS, N.B. 2005. Order Chiroptera. In Mammals Species of the World: a taxonomic and geographic reference. V. 1. (D.E. WILSON \& D.M. REEDER, eds.). Johns Hopkins University Press, Baltimore, p. 312-529.

TAVARES, V.C., GREGORIN, R. \& PERACCHI, A.L. no prelo. A diversidade de morcegos no Brasil. In Morcegos no Brasil: Biologia, Sistemática, Ecologia e Conservação (PACHECO, S.M., R.V. MARQUES \& C.E. ESBERARD, eds.). USEB, Pelotas.

VIZOTTO, L.D. \& TADDEI, V.A. 1973. Chave para a determinação de quirópteros brasileiros. Francal, São José do Rio Preto.

Título: Primeiro registro de Myotis albescens (É. Geoffroy, 1806) (Chiroptera, Vespertilionidae) para o Estado do Paraná, Brasil.

Autores: Miranda, JMD, Pulchério-Leite, A, Bernardi, IP, Passos, FC

Biota Neotropica, Vol.7 (number 1): 2007 http://www.biotaneotropica.org.br/v7n1/pt/abstract?shortcommunication+bn03407012007

Recebido em 20/06/06 - Versão reformulada recebida em 13/12/06 - Publicado em 28/03/07

ISSN 1676-0603 